\title{
Commentary: The Impact of Regional Astrocyte Interferon- $\gamma$ Signaling During Chronic Autoimmunity: A Novel Role for the Immunoproteasome
}

\author{
Brandon C. Smith ${ }^{1,2}$ \& Jessica L. Williams ${ }^{1 *}$ \\ 'Department of Neurosciences, Lerner Research Institute, Cleveland Clinic, Cleveland, $\mathrm{OH}$ \\ ${ }^{2}$ Department of Biological, Geological, and Environmental Sciences, Cleveland State University, Cleveland, $\mathrm{OH}$
}

Article Info

\section{Article Notes}

Received: January 06, 2021

Accepted: February 04, 2021

\section{*Correspondence:}

${ }^{*}$ Dr. Jessica L. Williams, Lerner Research Institute, Cleveland Clinic, 9500 Euclid Avenue/NC30, Cleveland, OH 44195; Tel: +1 216-445-3831; Fax: +1 216-444-7927; ORCID ID: 0000-00029801-9580; Email: williaj39@ccf.org.

(c) 2021 Williams JL. This article is distributed under the terms of the Creative Commons Attribution 4.0 International License.

\section{Keywords:}

Multiple sclerosis

Experimental autoimmune encephalomyelitis

Neuroinflammation

Astrocyte

Interferon-gamma

Immunoproteasome

\section{ABSTRACT}

Despite an increase in approved therapies for treating the inflammatory and neurodegenerative disease multiple sclerosis (MS), many of which have efficacy in the early, acute phases, there are no reliable treatments for the chronic, progressive stages of the disease. A deeper understanding of the biological underpinnings that govern differences between acute and chronic stages of MS and an animal model of MS, experimental autoimmune encephalomyelitis, will inform therapeutic development and personalized treatment strategies. It is well-known that the effects of inflammation are complex and the implications vary between stages. Complimentary to our recent publication, we will discuss here the pleiotropic effects of the cytokine interferony across disease states, along with the implications of downstream mechanisms of action.

MS is commonly thought of as an autoimmune, neurodegenerative disease of the central nervous system (CNS) ${ }^{1,2}$. Relapsing-remitting MS (RRMS) is a subtype of MS affecting $85 \%$ of patients and is characterized by periods of neurological dysfunction followed by partial or complete recovery ${ }^{2-4}$. The relapses in RRMS are typically associated with inflammation and the infiltration of immune cells into the CNS, and during remissions there is reduced inflammation ${ }^{4}$. Many patients with RRMS go on to develop secondary progressive MS (SPMS), in which there is enhanced brain atrophy and fewer remissions. Additionally, approximately $15 \%$ of patients develop primary progressive MS (PPMS), which is characterized by continuous neurological loss ${ }^{2,4}$. Both SPMS and PPMS have significantly dampened inflammation compared to RRMS 4 . However, all of the current FDAapproved therapies for MS are immunomodulatory ${ }^{5}$. As such, it is not surprising that they have little or no effect in SPMS and PPMS patients and in some cases have even led to patient worsening ${ }^{6-8}$. Effective treatments for SPMS and PPMS patients are desperately needed and further dissecting the molecular differences between these stages may provide critical insight.

Experimental autoimmune encephalomyelitis (EAE) is a commonly used animal model of MS, and like MS, EAE is inflammatory and progresses to include demyelination and neurodegeneration ${ }^{9}$. In EAE, T helper (Th) 1 and Th17 cells are primed against myelin by antigen-presenting cells and are then able to cross the blood-brain barrier $^{9,10}$. Once in the CNS parenchyma, $\mathrm{T}$ cells produce a variety of pro-inflammatory cytokines that signal directly to glia, including astrocytes, and can damage both myelin and axons ${ }^{9,11}$. Similar to MS, EAE induced in the $\mathrm{C} 57 \mathrm{Bl} / 6$ mouse strain has an acute, inflammatory 
stage and a chronic phase with altered and reduced inflammation ${ }^{2,9,12}$. While the role of Th1 cells in MS is more complicated, during EAE, they are the primary driver of clinical disease $\mathrm{e}^{9,13}$. Interferon (IFN) $\gamma$ is one of the primary cytokines that is produced by Th1 cells ${ }^{14}$. Traditionally, IFN $\gamma$ is thought of as pro-inflammatory, particularly during acute EAE; however, more recently, IFN $\gamma$ has been shown to have a protective role in chronic stages of both EAE and $\mathrm{MS}^{15-18}$. Early studies showed that while deleterious in acute stages, during chronic stages of MS, infusions of IFN $\gamma$ had little to no negative effect on clinical outcome ${ }^{19}$. Furthermore, in the MS marmoset model of MS, treatment with IFN $\gamma$ led to slower disease progression during chronic stages $^{20}$. Combined with EAE data demonstrating the differing effects of IFN $\gamma$ between stages, these findings suggest that while the pro-inflammatory effects of IFN $\gamma$ may be pathogenic during acute $\mathrm{MS}$, as the disease progresses, the protective functions of IFN $\gamma$ may help resolve the inflammatory damage seen in chronic MS.

IFN $\gamma$ differentially regulates many genes that may result in various inflammatory profiles between stages of neuroinflammation ${ }^{18}$; and yet, there is still much to be discovered about the specific downstream effects of IFN $\gamma$ signaling. We recently described a region- and stagespecific effect of IFN $\gamma$ on a protein complex called the immunoproteasome (iP) in astrocytes during EAE ${ }^{15}$. The iP is a specialized form of the constitutive proteasome and is strongly induced by IFN $\gamma^{20-22}$. Classically, the iP was described to process antigen for major histocompatibility complex (MHC) Class I-mediated presentation ${ }^{20-22}$. Since both EAE and MS are associated with MHC class II antigen presentation and astrocytes do not readily present antigens in vivo, we hypothesized that the astrocyte iP had alternative functions outside of antigen presentation ${ }^{22-25}$. Recently, it was demonstrated that the iP has a critical role in protein homeostasis, cellular proliferation, cytokine regulation, and the clearance of reactive oxygen species (ROS) ${ }^{15,26-28}$. We expanded on these findings, examining the effects of IFN $\gamma$ signaling and the iP in astrocytes during neuroinflammation. After confirming astrocytic iP expression in postmortem MS lesions, we found that increased iP expression correlated with a reduction in oxidative stress proteins in astrocytes, suggesting that the expression of the iP may be protective. Next, we induced EAE in $\mathrm{C} 57 \mathrm{Bl} / 6$ WT mice, and following the acute phase, mice were treated with a specific, global iP inhibitor. Upon treatment, clinical signs of EAE were immediately exacerbated. Similar to our findings in MS tissue, compared to vehicle-treated mice with EAE, lesions in mice treated with the iP inhibitor were larger, there was evidence of astrocyte dropout, and an increase in polyubiquitinated proteins and oxidative stress indicators in astrocytes. Together, these data suggested that the iP plays an important role in the clearance of damaged proteins and ROS, which enhances astrocyte survival and limits tissue damage. To show this was an astrocyte-specific phenomenon, we generated Ifngr $1^{\mathrm{fl} / \mathrm{fl}}$ Gfap-Cre mice and induced EAE. Compared to controls, the loss of astrocyte IFN $\gamma$ signaling resulted in reduced iP expression in astrocytes, exacerbated EAE, specifically in the later stages of the disease course, and an increase in lesion size, polyubiquitination, and oxidative stress.

Of note, Bassler and colleagues ${ }^{29}$ showed that inhibition of the iP during the induction of EAE led to an amelioration of disease. This was primarily attributed to the role of the iP in cytokine regulation in immune cell priming during acute EAE. Without the proper cytokine environment, autoreactive $\mathrm{T}$ cells may not be fully activated and thus would cause less damage. However, the stage of EAE is important to consider with regards to the function of the iP. Because immune cells are past the initial priming phase and there are fewer CNS infiltrating immune cells during chronic $\mathrm{EAE}^{9}$, the iP inhibitor-mediated cytokine dysregulation demonstrated by Bassler et al. during acute EAE likely plays a lesser role during chronic stages. We demonstrated a novel role for the iP in astrocytes during chronic EAE that does not directly involve infiltrating cells or antigen presentation, but rather focuses on restoration of cellular homeostasis.

Immunoproteasome inhibitors have been suggested as a potential treatment for $\mathrm{MS}^{30}$. Given the findings of Bassler et al., if translated to MS, iP inhibitors may reduce the activation of autoreactive $\mathrm{T}$ cells; however, this is akin to the immunomodulatory treatments already available. Further, MS pathology is different from EAE in that while EAE has a clear progression from acute to chronic stages, MS progression is more muddled ${ }^{4,5,9}$. Importantly, RRMS patients tend to have active lesions with many infiltrating cells with concurrent chronic active lesions that are less inflammatory $^{31}$. So, while iP inhibitors may reduce the formation of new lesions, they could simultaneously exacerbate chronic active lesions in RRMS by limiting mechanisms that maintain cellular homeostasis. In SPMS and PPMS, a majority of the lesions are chronic active and chronic inactive, which have few infiltrating cells ${ }^{31}$. As such, reducing the activation of immune cells using iP inhibitors would provide little or no benefit to SPMS and PPMS patients and may even cause additional damage by reducing the ability of CNS cells to clear ROS and damaged proteins, leading to further cell and tissue damage. In addition, since the iP is critical for antigen presentation, blockade of its function may lead to deleterious side effects or opportunistic infections stemming from immune system compromise. For these reasons, the use of iP inhibitors is ill-advised for all stages of MS.

Due to the reduced influence of the peripheral immune system during SPMS and PPMS, an agonist of the iP, or an 
inhibitor of an iP regulator, may prove beneficial in reducing CNS damage. Specifically, promotion of the proteome maintenance functions of the iP without induction of the pro-inflammatory components induced by IFN $\gamma$ is ideal. While there are currently no such agonists, there is some evidence of upstream iP inhibitors that could be evaluated as potential therapeutic targets ${ }^{32}$. Due to the differing pathological processes that underlie acute versus chronic MS and EAE, each stage should be examined and treated separately. Further analysis of stage-specific mechanisms ongoing during MS may yield specific treatment strategies and lead to great strides in our understanding of the disease.

\section{References}

1. Trapp BD, et al. Axonal transection in the lesions of multiple sclerosis. N Engl J Med 338, 278-285, doi: 10.1056/nejm199801293380502 (1998).

2. Trapp BD \& Nave KA. Multiple sclerosis: an immune or neurodegenerative disorder? Annual review of neuroscience 31, 247269, doi: 10.1146/annurev.neuro.30.051606.094313 (2008).

3. Filippi M, Rocca MA, Martino G, et al. Magnetization transfer changes in the normal appearing white matter precede the appearance of enhancing lesions in patients with multiple sclerosis. Ann Neurol 43 809-814, doi: 10.1002/ana.410430616 (1998).

4. Confavreux C, Vukusic S, Moreau T, et al. Relapses and progression of disability in multiple sclerosis. N Engl J Med 343, 1430-1438, doi: 10.1056/nejm200011163432001 (2000).

5. Miller DH \& Leary SM. Primary-progressive multiple sclerosis. The Lancet Neurology 6, 903- 912, doi: https://doi.org/10.1016/S14744422(07)70243-0 (2007).

6. Goodin DS, et al. Disease modifying therapies in multiple sclerosis: Subcommittee of the American Academy of Neurology and the MS Council for Clinical Practice Guidelines. Neurology 58, 169-178, doi: 10.1212/wnl.58.2.169 (2002).

7. Wingerchuk DM \& Carter JL. Multiple Sclerosis: Current and Emerging Disease-Modifying Therapies and Treatment Strategies. Mayo Clinic Proceedings 89, 225-240, doi: https://doi.org/10.1016/j. mayocp.2013.11.002 (2014).

8. Feinstein A, Freeman J \& Lo AC. Treatment of progressive multiple sclerosis: what works, what does not, and what is needed. The Lancet Neurology 14, 194-207, doi: https://doi.org/10.1016/S14744422(14)70231-5 (2015)

9. Constantinescu CS, Farooqi N, O’Brien K, et al. Experimental autoimmune encephalomyelitis (EAE) as a model for multiple sclerosis (MS). British journal of pharmacology 164, 1079-1106, doi: 10.1111/j.1476-5381.2011.01302.x (2011).

10. Hofstetter HH, Shive CL \& Forsthuber TG. Pertussis toxin modulates the immune response to neuroantigens injected in incomplete Freund's adjuvant: induction of Th1 cells and experimental autoimmune encephalomyelitis in the presence of high frequencies of Th2 cells. J Immunol 169, 117-125, doi: 10.4049/jimmunol.169.1.117 (2002).

11. Palle P, Monaghan KL, Milne SM, et al. Cytokine Signaling in Multiple Sclerosis and Its Therapeutic Applications. Med Sci (Basel) 5, 23, doi: 10.3390/medsci5040023 (2017).

12. Zorzella-Pezavento SFG, et al. Persistent inflammation in the CNS during chronic EAE despite local absence of IL-17 production. Mediators Inflamm 2013, 519627-519627, doi: $10.1155 / 2013 / 519627$ (2013)
13. Arellano G, et al. Th1 and Th17 Cells and Associated Cytokines Discriminate among Clinically Isolated Syndrome and Multiple Sclerosis Phenotypes. Frontiers in immunology 8, doi: 10.3389/ fimmu.2017.00753 (2017)

14. White RE, McTigue DM, Jakeman LB. Regional heterogeneity in astrocyte responses following contusive spinal cord injury in mice. Journal of Comparative Neurology 518, 1370- 1390, doi: 10.1002/ cne.22282 (2010).

15. Smith BC, Sinyuk M, Jenkins JE, et al. The impact of regional astrocyte interferon- $\gamma$ signaling during chronic autoimmunity: a novel role for the immunoproteasome. Journal of Neuroinflammation 17, 184, doi: 10.1186/s12974-020-01861-x (2020).

16. Hindinger C, et al. IFN- $\gamma$ Signaling to Astrocytes Protects from Autoimmune Mediated Neurological Disability. PLOS ONE 7, e42088, doi: 10.1371/journal.pone.0042088 (2012).

17. Arellano G, Ottum PA, Reyes LI, et al. Stage-Specific Role of InterferonGamma in Experimental Autoimmune Encephalomyelitis and Multiple Sclerosis. Frontiers in immunology 6, 492-492, doi: 10.3389/ fimmu.2015.00492 (2015).

18. Bever CTJr, Panitch HS, Levy HB, et al. Gamma-interferon induction in patients with chronic progressive MS. Neurology 41, 1124-1127, doi:10.1212/wnl.41.7.1124 (1991).

19. Jagessar SA, et al. Discrepant effects of human interferon-gamma on clinical and immunological disease parameters in a novel marmoset model for multiple sclerosis. J Neuroimmune Pharmacol 7, 253-265, doi: 10.1007/s11481-011-9320-5 (2012).

20. Murata S, Takahama Y, Kasahara M, et al. The immunoproteasome and thymoproteasome: functions, evolution and human disease. Nature Immunology 19, 923-931, doi:10.1038/s41590-018-0186-z (2018).

21. Ferrington DA \& Gregerson DS. Immunoproteasomes: structure, function, and antigen presentation. Prog Mol Biol Transl Sci 109, 75112, doi: 10.1016/B978-0-12-397863-9.00003-1 (2012).

22. Riedhammer C \& Weissert R. Antigen Presentation, Autoantigens, and Immune Regulation in Multiple Sclerosis and Other Autoimmune Diseases. Frontiers in immunology 6, doi: 10.3389/fimmu.2015.00322 (2015).

23. Traugott U. Multiple sclerosis: relevance of Class I and Class II MHCexpressing cells to lesion development. Journal of Neuroimmunology 16, 283-302, doi: https://doi.org/10.1016/0165- 5728(87)90082-8 (1987).

24. Sosa RA, Murphey C, Ji N, et al. The Kinetics of Myelin Antigen Uptake by Myeloid Cells in the Central Nervous System during Experimental Autoimmune Encephalomyelitis. The Journal of Immunology 191, 5848-5857, doi: 10.4049/jimmunol.1300771 (2013).

25. Bo L, et al. Detection of MHC class II-antigens on macrophages and microglia, but not on astrocytes and endothelia in active multiple sclerosis lesions. J Neuroimmunol 51, 135-146 (1994).

26. Seifert U, et al. Immunoproteasomes Preserve Protein Homeostasis upon Interferon-Induced Oxidative Stress. Cell 142, 613-624, doi: https://doi.org/10.1016/j.cell.2010.07.036 (2010).

27. Kimura H, Caturegli P, Takahashi $M$, et al. New Insights into the Function of the Immunoproteasome in Immune and Nonimmune Cells. J Immunol Res 2015, 541984-541984, doi: 10.1155/2015/541984 (2015).

28. Ebstein F, Kloetzel PM, Krüger E, et al. Emerging roles of immunoproteasomes beyond MHC class I antigen processing. Cellular and Molecular Life Sciences 69, 2543-2558, doi: 10.1007/s00018012-0938-0 (2012).

29. Basler $M$, et al. Inhibition of the immunoproteasome ameliorates experimental autoimmune encephalomyelitis. EMBO molecular medicine 6, 226-238, doi: 10.1002/emmm.201303543 (2014). 
30. Bellavista E, et al. Current Understanding on the Role of Standard and Immunoproteasomes in Inflammatory/Immunologica Pathways of Multiple Sclerosis. Autoimmune Diseases 2014, 12, doi: 10.1155/2014/739705 (2014).

31. Dutta R \& Trapp BD. Relapsing and progressive forms of multiple sclerosis: insights from pathology. Curr Opin Neurol 27, 271-278, doi: 10.1097/WCO.0000000000000094 (2014).

32. Anderson AM, et al. The metastasis suppressor RARRES3 as an endogenous inhibitor of the immunoproteasome expression in breast cancer cells. Scientific reports 7, 39873, doi:10.1038/srep39873 (2017). 